\title{
Correction to: Use of GMDH-type neural network to model the mechanical behavior of a cement-treated sand
}

\author{
Hossein MolaAbasi ${ }^{1} \cdot{\text { Aghileh } \text { Khajeh }^{2} \text { (1) } \cdot \text { Reza Jamshidi Chenari }}^{2}$
}

Received: 25 September 2021 / Accepted: 25 September 2021 / Published online: 29 October 2021

(c) Springer-Verlag London Ltd., part of Springer Nature 2021

\section{Correction to: Neural Computing and Applications} https://doi.org/10.1007/s00521-021-06157-6

Unfortunately, the co-author name (Reza Jamshidi Chenari) has been incorrectly mentioned as co-corresponding author along with the incorrect e-mail address (aqilekhajeh@phd.guilan.ac.ir).
The correct details are provided in the author group section.

Publisher's Note Springer Nature remains neutral with regard to jurisdictional claims in published maps and institutional affiliations.

The original article can be found online at https:// doi.org/10.1007/s00521-021-06157-6.

Aghileh Khajeh

aqilekhajeh@phd.guilan.ac.ir

1 Department of Civil Engineering, Gonbad Kavous University, Gonbad Kavous, Golestan, Iran

2 Department of Civil Engineering, University of Guilan, Rasht, Guilan, Iran 